\title{
ON A NONLOCAL DIFFERENTIAL EQUATION DESCRIBING ROOTS OF POLYNOMIALS UNDER DIFFERENTIATION
}

\author{
RAFAEL GRANERO-BELINCHÓN
}

\begin{abstract}
In this work we study the nonlocal transport equation derived recently by Steinerberger when studying how the distribution of roots of a polynomial behaves under iterated differentation of the function. In particular, we study the well-posedness of the equation, establish some qualitative properties of the solution and give conditions ensuring the global existence of both weak and strong solutions. Finally, we present a link between the equation obtained by Steinerberger and a one-dimensional model of the surface quasi-geostrophic equation used by Chae, Córdoba, Córdoba \& Fontelos.
\end{abstract}

\section{INTRODUCTION AND MAIN RESULTS}

In this paper, we consider the following one-dimensional nonlinear transport equation

$$
\partial_{t} u+\partial_{x} \arctan \left(\frac{H u}{u}\right)=0 \quad(x, t) \text { on } \mathbb{S}^{1} \times[0, T],
$$

where

$$
H u(x)=\frac{1}{2 \pi} \text { p.v. } \int_{\mathbb{S}} \frac{u(y)}{\tan \left(\frac{x-y}{2}\right)} d y,
$$

is the Hilbert transform. The previous equation needs to be supplemented with the initial data

$$
u(x, 0)=u_{0}(x) .
$$

This equation has been derived by S. Steinerberger [31] when studying how the distribution of roots behaves under iterated differentation. In particular, the purpose of this work is to study the properties of the transport equation (1). The study of such nonlocal and nonlinear one-dimensional equations is a wide research area with a large literature. For other similar equations and related results we refer to $[4,5,8,12,13,22,14,15,23,25$, $28,24]$.

In this paper we prove the following results:

Date: December 4, 2018. 
Theorem 1. Let $0<u_{0} \in H^{2}\left(\mathbb{S}^{1}\right)$ be the initial data. Then there exists a time $0<T \leqslant \infty, T=T\left(\left\|u_{0}\right\|_{H^{2}}, \min _{x} u_{0}(x)\right)$ and a unique positive solution to $(1)$

$$
0<u \in C\left([0, T], H^{2}\left(\mathbb{S}^{1}\right)\right) .
$$

Furthermore, this solution verifies the following properties:

$$
\|u(t)\|_{L^{2}}^{2}+\int_{0}^{t} \mathcal{D}(s) d s=\left\|u_{0}\right\|_{L^{2}}^{2}
$$

where

$\mathcal{D}=\frac{1}{16 \pi} \int_{\mathbb{R}} p \cdot v \cdot \int_{\mathbb{S}} \frac{u(x)-u(y)}{\sin \left(\frac{x-y}{2}\right)^{2}} \log \left(\frac{u(x)^{2}+(H u(x))^{2}}{u(y)^{2}+(H u(y))^{2}}\right) d x d y$,

- $\|u(t)\|_{L^{1}}=\left\|u_{0}\right\|_{L^{1}}$,

- if $u_{0}(x)$ is even, $u(x, t)$ remains even,

- Maximum principle: $\max _{x} u(x, t) \leqslant \max _{x} u_{0}(x)$,

- Minimum principle: $\min _{x} u_{0}(x) \leqslant \min _{x} u(x, t)$.

Remark 1. We remark that, for an arbitrary $u(x, t)$, we are not able to give a sign to $\mathcal{D}$ (compare with $[1,17])$. In other words, we are not able to show whether the $L^{2}$ norm decays.

Remark 2. The following scaling is invariant for the equation $u_{\lambda}(x, t)=$ $\lambda^{\alpha} u\left(\lambda x, \lambda^{1-\alpha} t\right) \forall \alpha \in \mathbb{R}$.

Under certain restrictions we can ensure that the solution is global:

Theorem 2. Let $0<u_{0} \in H^{2}\left(\mathbb{S}^{1}\right)$ be the initial data and denote

$$
\left\langle u_{0}\right\rangle=\frac{1}{2 \pi} \int_{\mathbb{S}^{1}} u_{0}(x) d x .
$$

There exists $0<\mathcal{C}$ such that if

$$
\frac{\left\|u_{0}-\left\langle u_{0}\right\rangle\right\|_{A^{1}}}{\left\langle u_{0}\right\rangle} \leqslant \mathcal{C}
$$

then the solution (from Theorem 1) is global and satisfies

$$
\left\|u(t)-\left\langle u_{0}\right\rangle\right\|_{A^{1}} \leqslant\left\|u_{0}-\left\langle u_{0}\right\rangle\right\|_{A^{1}} e^{-\delta t}
$$

for certain $0<\delta\left(\left\langle u_{0}\right\rangle\right)$ small enough.

Remark 3. The explicit lower bound $0.17<\mathcal{C}$ is obtained as a byproduct.

Finally, we study the existence of weak solutions i.e. solutions that satisfy the equation in the following sense:

$-\int_{0}^{T} \int_{\mathbb{S}^{1}} u(x, s) \partial_{t} \phi(x, s)+\arctan \left(\frac{H u(x, s)}{u(x, s)}\right) \partial_{x} \phi d x d s=\int_{\mathbb{S}^{1}} u_{0}(x) \phi(x, 0) d x$,

for all test functions $\phi(x, t) \in C^{\infty}\left(\mathbb{S}^{1} \times[0, T)\right)$. 
In that regards, we prove the global existence of weak solution for initial data satisfying certain size conditions in a scale invariant space (with respect to the scaling of the equation):

Theorem 3. Let $0<u_{0} \in A^{0}\left(\mathbb{S}^{1}\right)$ be the initial data and denote

$$
\left\langle u_{0}\right\rangle=\frac{1}{2 \pi} \int_{\mathbb{S}^{1}} u_{0}(x) d x .
$$

There exists $0<\tilde{\mathcal{C}}$ such that if

$$
\frac{\left\|u_{0}-\left\langle u_{0}\right\rangle\right\|_{A^{0}}}{\left\langle u_{0}\right\rangle} \leqslant \tilde{\mathcal{C}}
$$

then there exists at least one global weak solution

$$
u \in L^{\infty}\left([0, T] \times \mathbb{S}^{1}\right) \cap L^{2}\left(0, T ; H^{0.5}\right), \forall 0<T<\infty
$$

and this solution satisfies

$$
\left\|u(t)-\left\langle u_{0}\right\rangle\right\|_{L^{\infty}} \leqslant\left\|u_{0}-\left\langle u_{0}\right\rangle\right\|_{A^{0}} e^{-\delta t}
$$

for certain $0<\delta\left(\left\langle u_{0}\right\rangle\right)$ small enough.

Remark 4. The explicit lower bound $0.24<\tilde{\mathcal{C}}$ is obtained as a byproduct.

Remark 5. Similar results can be proved for the porous medium equation (see also [30, 29])

$$
\partial_{t} u+\partial_{x}\left(\frac{H u}{u^{m}}\right)=0, m \in \mathbb{N} .
$$

The rest of the paper is devoted to the proofs of each results (sections 2-4) and the link between (1) and the equation

$$
\partial_{t} g+\Lambda g=\partial_{x}(g H g) \text {, }
$$

(see section 5). We would like to remark that (4) was proposed as a onedimensional model of the 2D surface quasi-geostrophic equation by Chae, Córdoba, Córdoba \& Fontelos [6] (see also the papers by Matsuno [27] and Baker, Li \& Morlet [2]).

Notation. We denote

$$
\Lambda u=H \partial_{x} u(x)=\frac{1}{4 \pi} \text { p.v. } \int_{\mathbb{S}} \frac{u(x)-u(x-y)}{\sin ^{2}(y / 2)} d y .
$$

We define the $L^{2}$ based Sobolev spaces

$$
H^{s}=\left\{u(x)=\sum_{n \in \mathbb{Z}} \hat{u}(n) e^{i n x} \text { with } \sum_{n \in \mathbb{Z}}|n|^{2 s}|\hat{u}(n)|^{2}<\infty\right\},
$$

with norm $\|u\|_{H^{s}}=\left\|\Lambda^{s} u\right\|_{L^{2}}$. Similarly, we recall the definition of the Wiener spaces

$$
A^{s}=\left\{u(x)=\sum_{n \in \mathbb{Z}} \hat{u}(n) e^{i n x} \text { with } \sum_{n \in \mathbb{Z}}|n|^{s}|\hat{u}(n)|<\infty\right\} .
$$


with norm $\|u\|_{A^{s}}=\left\|\widehat{\Lambda^{s} u}\right\|_{\ell^{1}}$.

\section{Proof of Theorem 1}

Well-posedness. The existence will follow using the energy method [26] once the appropriate a priori estimates are obtained. We define the energy

$$
\mathcal{E}(t)=\frac{1}{\min _{x} u(x, t)}+\|u(t)\|_{H^{2}} .
$$

We have to proof an inequality of the type

$$
\frac{d}{d t} \mathcal{E}(t) \leqslant C(1+\mathcal{E}(t))^{p}
$$

for certain $C$ and $p$.

To estimate the first term in the energy we use a pointwise argument (see $[11,9,19,3]$ for more details). The solution has at least a minimum:

$$
m(t)=\min _{x} u(x, t)=u\left(\underline{x}_{t}, t\right) .
$$

Because of the positivity of the initial data, we have that $m(0)>0$. Following the argument in $[11,19,3]$, we have that

$$
\frac{d}{d t} m(t)=\partial_{t} u\left(\underline{x}_{t}, t\right)=-\frac{m(t) \Lambda u\left(\underline{x}_{t}\right)}{m(t)^{2}+\left(H u\left(\underline{x}_{t}\right)\right)^{2}} \text { a.e.. }
$$

Then,

$$
\frac{d}{d t} \frac{1}{\min _{x} u(x, t)}=-\frac{\partial_{t} u\left(\underline{x}_{t}, t\right)}{m(t)^{2}} \leqslant C \frac{\|u\|_{H^{2}}}{m(t)^{3}} \leqslant C(\mathcal{E}(t))^{4}
$$

For the sake of brevity we only provide with the estimates for the higher order terms (being the rest of the terms straightforward). We take 2 derivatives of the equation and test against $\partial_{x}^{2} u$. We find that

$$
\begin{aligned}
\frac{d}{d t}\|u(t)\|_{\dot{H}^{2}}^{2}= & -\frac{\left(\partial_{x}^{2} u \Lambda u+u \Lambda \partial_{x}^{2} u\right) \partial_{x}^{2} u}{u^{2}+(H u)^{2}}+\frac{u \Lambda u\left(2 u \partial_{x}^{2} u+2 H u \partial_{x} \Lambda u\right) \partial_{x}^{2} u}{\left(u^{2}+(H u)^{2}\right)^{2}} \\
& +\frac{\left(\partial_{x} \Lambda u \partial_{x} u+H u \partial_{x}^{3} u\right) \partial_{x}^{2} u}{u^{2}+(H u)^{2}}-\frac{H u \partial_{x} u\left(2 u \partial_{x}^{2} u+2 H u \Lambda \partial_{x} u\right) \partial_{x}^{2} u}{\left(u^{2}+(H u)^{2}\right)^{2}}+\text { l.o.t. } \\
= & I_{1}+I_{2}+I_{3}+I_{4}+\text { l.o.t.. }
\end{aligned}
$$

Using that

$$
\left\|\frac{1}{u^{2}+(H u)^{2}}\right\|_{L^{\infty}} \leqslant\left\|\frac{1}{u^{2}}\right\|_{L^{\infty}} \leqslant \frac{1}{m(t)^{2}} \leqslant \mathcal{E}(t)^{2},
$$

we have that

$$
\text { l.o.t. } \leqslant C(\mathcal{E}(t))^{8}\|u(t)\|_{\dot{H}^{2}} \text {. }
$$

We recall the Córdoba-Córdoba inequality [10]

$$
\theta \Lambda \theta \geqslant \frac{1}{2} \Lambda\left(\theta^{2}\right)
$$


to find that

$$
\begin{gathered}
\int_{\mathbb{S}^{1}} \frac{u \Lambda \partial_{x}^{2} u \partial_{x}^{2} u}{u^{2}+(H u)^{2}} d x \geqslant \frac{1}{2} \int_{\mathbb{S}^{1}} H \partial_{x}\left(\frac{u}{u^{2}+(H u)^{2}}\right)\left(\partial_{x}^{2} u\right)^{2} d x \\
I_{1} \leqslant \frac{\|u(t)\|_{\dot{H}^{2}}^{2}\|u\|_{A^{1}}}{m(t)^{2}}-\frac{1}{2} \int_{\mathbb{S}^{1}} H \partial_{x}\left(\frac{u}{u^{2}+(H u)^{2}}\right)\left(\partial_{x}^{2} u\right)^{2} d x \\
\leqslant \frac{\|u(t)\|_{\dot{H}^{2}}^{2}\|u\|_{A^{1}}}{m(t)^{2}}+\frac{1}{2} \frac{\|u(t)\|_{\dot{H}^{2}}^{2}\|u\|_{A^{1}}}{m(t)^{2}}+\frac{\|u(t)\|_{\dot{H}^{2}}^{2}\|u\|_{A^{0}}^{2}\|u\|_{A^{1}}}{m(t)^{4}} \\
\leqslant C(1+\mathcal{E}(t))^{8}\|u(t)\|_{\dot{H}^{2}} .
\end{gathered}
$$

A similar use of Hölder inequality and Sobolev embedding leads to

$$
I_{2}+I_{4} \leqslant C(1+\mathcal{E}(t))^{8}\|u(t)\|_{\dot{H}^{2}} .
$$

Finally, we observe that an integration by parts allow us to conclude

$$
I_{3} \leqslant C(1+\mathcal{E}(t))^{8}\|u(t)\|_{\dot{H}^{2}} .
$$

Then, we obtain that

$$
\frac{1}{2} \frac{d}{d t}\|u(t)\|_{\dot{H}^{2}}^{2} \leqslant C(1+\mathcal{E}(t))^{8}\|u(t)\|_{\dot{H}^{2}} .
$$

Thus, collecting (6),(7) and (8), we conclude te desired inequality (5)

An identity for the evolution of the $L^{2}$ norm. We test (1) against $u$. We find that

$$
\frac{d}{d t}\|u(t)\|_{L^{2}}^{2}=\int_{\mathbb{S}^{1}}-\frac{u \Lambda u u}{u^{2}+(H u)^{2}}+\frac{H u \partial_{x} u u}{u^{2}+(H u)^{2}} d x
$$

We compute

$$
\begin{aligned}
\mathcal{D} & =-\frac{1}{2} \int_{\mathbb{S}^{1}} H u \partial_{x} \log \left(u^{2}+(H u)^{2}\right) d x \\
& =-\int_{\mathbb{S}^{1}} H u \frac{u \partial_{x} u+H u \Lambda u}{u^{2}+(H u)^{2}} d x \\
& =-\int_{\mathbb{S}^{1}} \frac{u \partial_{x} u H u}{u^{2}+(H u)^{2}}-\frac{u^{2} \Lambda u}{u^{2}+(H u)^{2}}+\Lambda u d x \\
& =-\int_{\mathbb{S}^{1}} \frac{u \partial_{x} u H u}{u^{2}+(H u)^{2}}-\frac{u^{2} \Lambda u}{u^{2}+(H u)^{2}} d x .
\end{aligned}
$$

As a consequence,

Furthermore,

$$
\frac{d}{d t}\|u(t)\|_{L^{2}}^{2}=-\mathcal{D}
$$

$$
\begin{aligned}
\mathcal{D} & =\frac{1}{2} \int_{\mathbb{S}^{1}} \Lambda u \log \left(u^{2}+(H u)^{2}\right) d x \\
& =\frac{1}{8 \pi} \int_{\mathbb{S}^{1}} \text { p.v. } \int_{\mathbb{S}} \frac{u(x)-u(x-y)}{\sin (y / 2)^{2}} \log \left(u(x)^{2}+(H u(x))^{2}\right) d x d y
\end{aligned}
$$




$$
\begin{aligned}
& =\frac{1}{8 \pi} \int_{\mathbb{S}^{1}} \text { p.v. } \int_{\mathbb{S}} \frac{u(x)-u(y)}{\sin ((x-y) / 2)^{2}} \log \left(u(x)^{2}+(H u(x))^{2}\right) d x d y \\
& =\frac{1}{8 \pi} \int_{\mathbb{S}^{1}} \text { p.v. } \int_{\mathbb{S}} \frac{u(y)-u(x)}{\sin ((x-y) / 2)^{2}} \log \left(u(y)^{2}+(H u(y))^{2}\right) d x d y .
\end{aligned}
$$

Then, we have identity (3). This concludes with the existence part. The uniqueness follow from a standard contradiction argument.

Propagation of the $L^{1}$ norm. Once the solution remains positive, the $L^{1}$ norm is preserved due to the divergence form of the equation.

Propagation of the even symmetry. This is a straightforward consequence of the fact that the Hilbert transform $H$ maps even functions into odd functions.

Maximum principle. We define

$$
M(t)=\max _{x} u(x, t)=u\left(\bar{x}_{t}, t\right)
$$

Then (see $[16,19,3]$ for more details) we have that

$$
\frac{d}{d t} M(t)=\partial_{t} u\left(\bar{x}_{t}, t\right) \text { a.e. }
$$

Then

We observe that

$$
\frac{d}{d t} M(t)+\frac{M(t) \Lambda u\left(\bar{x}_{t}\right)}{M(t)^{2}+\left(H u\left(\bar{x}_{t}\right)\right)^{2}}=0
$$

$$
\Lambda u\left(\bar{x}_{t}\right) \geqslant 0
$$

Thus, using $0 \leqslant M(t)$, we obtain that

$$
M(t) \leqslant M(0)
$$

Minimum principle. With the previous definition for $m(t)$, we have that

$$
\frac{d}{d t} m(t)+\frac{m(t) \Lambda u\left(\underline{x}_{t}\right)}{m(t)^{2}+\left(H u\left(\underline{x}_{t}\right)\right)^{2}}=0 .
$$

Thus, using $\Lambda u\left(\underline{x}_{t}\right) \leqslant 0$, we find that

$$
0 \leqslant m(0) \leqslant m(t)
$$

\section{Proof of Theorem 2}

The proof of this Theorem follows the approach in [18]. We define the new variable

$$
v(x, t)=u(x, t)-\left\langle u_{0}\right\rangle .
$$

This variable quantifies the difference between the steady state $u_{\infty}=\left\langle u_{0}\right\rangle$ and $u$. The idea of the theorem is first to linearize around the stady state $\left\langle u_{0}\right\rangle$. Secondly, we obtain an inequality of the form

$$
\frac{d}{d t}\|v(t)\|_{A^{1}}+\frac{\|v(t)\|_{A^{2}}}{\left\langle u_{0}\right\rangle} \leqslant \mathcal{F}\left(\frac{\|v(t)\|_{A^{1}}}{\left\langle u_{0}\right\rangle}\right)\|v(t)\|_{A^{2}}
$$


with $\mathcal{F}(0)=0$ and $\mathcal{F}$ continuous. We observe that this inequality guarantees $v(t) \rightarrow 0$ in $A^{1}$ for small enough $\left\|v_{0}\right\|_{A^{1}} /\left\langle u_{0}\right\rangle$.

In what follows we assume that

$$
r=\frac{\|v\|_{A^{1}}}{\left\langle u_{0}\right\rangle}<\frac{1}{2}
$$

so that

$$
\frac{\|v\|_{A^{1}}}{\left\langle u_{0}\right\rangle-\|v\|_{A^{1}}}=\frac{r}{1-r}<1
$$

Since we have the following Poincaré type inequality

$$
\|v\|_{A^{s}} \leqslant\|v\|_{A^{r}}, \forall 0 \leqslant s<r
$$

we observe that

$$
\left|\frac{H u}{u}\right|=\left|\frac{H v}{u}\right| \leqslant \frac{\|v\|_{A^{0}}}{\left\langle u_{0}\right\rangle-\|v\|_{A^{0}}} \leqslant \frac{\|v\|_{A^{1}}}{\left\langle u_{0}\right\rangle-\|v\|_{A^{1}}}<1 .
$$

As a consequence, we can expand the nonlinearity as a power series

$$
\arctan \left(\frac{H u}{u}\right)=\sum_{n \in \mathbb{Z}^{+} \cup\{0\}} \frac{(-1)^{n}}{1+2 n}\left(\frac{H u}{u}\right)^{1+2 n},
$$

SO

$$
\partial_{t} u=-\sum_{n \in \mathbb{Z}^{+} \cup\{0\}}(-1)^{n}\left(\frac{H u}{u}\right)^{2 n}\left(\frac{\Lambda u}{u}-\frac{H u \partial_{x} u}{u^{2}}\right) .
$$

In the new variable, this latter equation reads

$$
\begin{aligned}
\partial_{t} v= & -\sum_{n \in \mathbb{Z}^{+}}(-1)^{n}\left(\frac{H v}{v+\left\langle u_{0}\right\rangle}\right)^{2 n}\left(\frac{\Lambda v}{v+\left\langle u_{0}\right\rangle}-\frac{H v \partial_{x} v}{\left(v+\left\langle u_{0}\right\rangle\right)^{2}}\right) \\
& -\left(\frac{\Lambda v}{v+\left\langle u_{0}\right\rangle}-\frac{H v \partial_{x} v}{\left(v+\left\langle u_{0}\right\rangle\right)^{2}}\right) .
\end{aligned}
$$

We recall the following Taylor series

$$
\begin{gathered}
\frac{1}{\left\langle u_{0}\right\rangle+v}=\frac{1}{\left\langle u_{0}\right\rangle}+\frac{1}{\left\langle u_{0}\right\rangle} \sum_{n \in \mathbb{Z}^{+}}(-1)^{n}\left(\frac{v}{\left\langle u_{0}\right\rangle}\right)^{n}, \\
\frac{1}{\left(\left\langle u_{0}\right\rangle+v\right)^{2}}=\frac{1}{\left\langle u_{0}\right\rangle^{2}}+\frac{1}{\left\langle u_{0}\right\rangle^{2}} \sum_{n \in \mathbb{Z}^{+}}(-1)^{n}(1+n)\left(\frac{v}{\left\langle u_{0}\right\rangle}\right)^{n} .
\end{gathered}
$$

We define

$$
\begin{aligned}
& \mathfrak{S}_{1}=\frac{H v}{\left\langle u_{0}\right\rangle}+\frac{H v}{\left\langle u_{0}\right\rangle} \sum_{m \in \mathbb{Z}^{+}}(-1)^{m}\left(\frac{v}{\left\langle u_{0}\right\rangle}\right)^{m} \\
& \mathfrak{S}_{2}=\frac{H v \partial_{x} v}{\left\langle u_{0}\right\rangle^{2}}+\frac{H v \partial_{x} v}{\left\langle u_{0}\right\rangle^{2}} \sum_{m \in \mathbb{Z}^{+}}(-1)^{m}(1+m)\left(\frac{v}{\left\langle u_{0}\right\rangle}\right)^{m} \\
& \mathfrak{S}_{3}=\frac{\Lambda v}{\left\langle u_{0}\right\rangle} \sum_{m \in \mathbb{Z}^{+}}(-1)^{m}\left(\frac{v}{\left\langle u_{0}\right\rangle}\right)^{m} .
\end{aligned}
$$


Using the previous Taylor series together with the previous definitions, we find that

$$
\begin{aligned}
\partial_{t} v+\frac{\Lambda v}{\left\langle u_{0}\right\rangle}= & -\sum_{n \in \mathbb{Z}^{+}}(-1)^{n}\left(\mathfrak{S}_{1}\right)^{2 n}\left(\frac{\Lambda v}{\left\langle u_{0}\right\rangle}+\mathfrak{S}_{3}\right) \\
& +\sum_{n \in \mathbb{Z}^{+}}(-1)^{n}\left(\mathfrak{S}_{1}\right)^{2 n} \mathfrak{S}_{2}-\mathfrak{S}_{3}+\mathfrak{S}_{2} .
\end{aligned}
$$

We take a derivative of (9) to obtain that

$$
\begin{aligned}
\partial_{t} \partial_{x} v+\frac{\Lambda \partial_{x} v}{\left\langle u_{0}\right\rangle}= & \sum_{n \in \mathbb{Z}^{+}}(-1)^{n+1} 2 n\left(\mathfrak{S}_{1}\right)^{2 n-1} \partial_{x} \mathfrak{S}_{1}\left(\frac{\Lambda v}{\left\langle u_{0}\right\rangle}+\mathfrak{S}_{3}\right) \\
& -\sum_{n \in \mathbb{Z}^{+}}(-1)^{n}\left(\mathfrak{S}_{1}\right)^{2 n}\left(\frac{\Lambda \partial_{x} v}{\left\langle u_{0}\right\rangle}+\partial_{x} \mathfrak{S}_{3}\right) \\
& +\sum_{n \in \mathbb{Z}^{+}}(-1)^{n} 2 n\left(\mathfrak{S}_{1}\right)^{2 n-1} \partial_{x} \mathfrak{S}_{1} \mathfrak{S}_{2}+\sum_{n \in \mathbb{Z}^{+}}(-1)^{n}\left(\mathfrak{S}_{1}\right)^{2 n} \partial_{x} \mathfrak{S}_{2} \\
& -\partial_{x} \mathfrak{S}_{3}+\partial_{x} \mathfrak{S}_{2}
\end{aligned}
$$

$$
\|v\|_{A^{1}}=\left\|\partial_{x} v\right\|_{A^{0}}
$$

To do that we first observe that $A^{0}$ is an algebra, thus,

$$
\left\|\mathfrak{S}_{1}^{2 n}\right\|_{A^{0}} \leqslant\left\|\mathfrak{S}_{1}\right\|_{A^{0}}^{2 n} \leqslant\left(\frac{\|v\|_{A^{0}}}{\left\langle u_{0}\right\rangle}+\frac{\|v\|_{A^{0}}}{\left\langle u_{0}\right\rangle} \sum_{m \in \mathbb{Z}^{+}}\left(\frac{\|v\|_{A^{0}}}{\left\langle u_{0}\right\rangle}\right)^{m}\right)^{2 n} .
$$

Summing up the series, we find the estimate

$$
\left\|\mathfrak{S}_{1}^{2 n}\right\|_{A^{0}} \leqslant\left(\frac{\|v\|_{A^{0}}}{\left\langle u_{0}\right\rangle-\|v\|_{A^{0}}}\right)^{2 n}
$$

Similarly,

$$
\begin{aligned}
\left\|\mathfrak{S}_{1}^{2 n-1}\right\|_{A^{0}} & \leqslant\left(\frac{\|v\|_{A^{0}}}{\left\langle u_{0}\right\rangle-\|v\|_{A^{0}}}\right)^{2 n-1} \\
\left\|\mathfrak{S}_{2}\right\|_{A^{0}} & \leqslant \frac{\|v\|_{A^{0}}\|v\|_{A^{1}}}{\left\langle u_{0}\right\rangle^{2}}+\frac{\|v\|_{A^{0}}\|v\|_{A^{1}}}{\left\langle u_{0}\right\rangle^{2}} \sum_{m \in \mathbb{Z}^{+}}(1+m)\left(\frac{\|v\|_{A^{0}}}{\left\langle u_{0}\right\rangle}\right)^{m} \leqslant \frac{\|v\|_{A^{0}}\|v\|_{A^{1}}}{\left(\left\langle u_{0}\right\rangle-\|v\|_{A^{0}}\right)^{2}} \\
\left\|\mathfrak{S}_{3}\right\|_{A^{0}} & \leqslant \frac{\|v\|_{A^{1}}}{\left\langle u_{0}\right\rangle} \sum_{m \in \mathbb{Z}^{+}}\left(\frac{\|v\|_{A^{0}}}{\left\langle u_{0}\right\rangle}\right)^{m} \leqslant \frac{\|v\|_{A^{1}}\|v\|_{A^{0}}}{\left\langle u_{0}\right\rangle\left(\left\langle u_{0}\right\rangle-\|v\|_{A^{0}}\right)} \\
\left\|\partial_{x} \mathfrak{S}_{1}\right\|_{A^{0}} & \leqslant \frac{\|v\|_{A^{1}}}{\left\langle u_{0}\right\rangle-\|v\|_{A^{0}}}+\frac{\|v\|_{A^{1}}\|v\|_{A^{0}}}{\left(\left\langle u_{0}\right\rangle-\|v\|_{\left.A^{0}\right)^{2}}\right.} \\
\left\|\partial_{x} \mathfrak{S}_{2}\right\|_{A^{0}} & \leqslant \frac{\|v\|_{A^{1}}^{2}+\|v\|_{A^{0}}\|v\|_{A^{2}}}{\left(\left\langle u_{0}\right\rangle-\|v\|_{A^{0}}\right)^{2}}+\frac{2\|v\|_{A^{0}}\|v\|_{A^{1}}^{2}}{\left(\left\langle u_{0}\right\rangle-\|v\|_{A^{0}}\right)^{3}} \\
\left\|\partial_{x} \mathfrak{S}_{3}\right\|_{A^{0}} & \leqslant \frac{\|v\|_{A^{2}}\|v\|_{A^{0}}}{\left\langle u_{0}\right\rangle\left(\left\langle u_{0}\right\rangle-\|v\|_{A^{0}}\right)}+\frac{\|v\|_{A^{1}}^{2}}{\left(\left\langle u_{0}\right\rangle-\|v\|_{A^{0}}\right)^{2}} .
\end{aligned}
$$


We obtain that

$$
\begin{aligned}
\frac{d}{d t}\|v\|_{A^{1}}+\frac{\|v\|_{A^{2}}}{\left\langle u_{0}\right\rangle} \leqslant & \frac{\|v\|_{A^{2}}}{\left\langle u_{0}\right\rangle}\left\{\sum_{n \in \mathbb{Z}^{+}} 2 n\left(\frac{r}{1-r}\right)^{2 n-1}\left[\frac{r}{1-r}+\frac{r^{2}}{(1-r)^{2}}\right] \frac{1}{1-r}\right. \\
& +\sum_{n \in \mathbb{Z}^{+}}\left(\frac{r}{1-r}\right)^{2 n}\left(1+\frac{r}{1-r}+\frac{r}{(1-r)^{2}}\right) \\
& +\sum_{n \in \mathbb{Z}^{+}} 2 n\left(\frac{r}{1-r}\right)^{2 n-1}\left[\frac{r}{1-r}+\frac{r^{2}}{(1-r)^{2}}\right] \frac{r}{(1-r)^{2}} \\
& +2 \sum_{n \in \mathbb{Z}^{+}}\left(\frac{r}{1-r}\right)^{2 n}\left(\frac{r}{(1-r)^{2}}+\frac{r^{2}}{(1-r)^{3}}\right) \\
& \left.+\frac{r}{1-r}+\frac{r}{(1-r)^{2}}\right\} .
\end{aligned}
$$

Using

$$
\frac{z^{2}}{1-z^{2}}=\sum_{n \in \mathbb{Z}^{+}} z^{2 n},
$$

we find

$$
\begin{aligned}
\frac{d}{d t}\|v\|_{A^{1}}+\frac{\|v\|_{A^{2}}}{\left\langle u_{0}\right\rangle} \leqslant & \frac{\|v\|_{A^{2}}}{\left\langle u_{0}\right\rangle}\left\{\frac{2 \frac{r}{1-r}}{\left(1-\left(\frac{r}{1-r}\right)^{2}\right)^{2}}\left[\frac{r}{1-r}+\frac{r^{2}}{(1-r)^{2}}\right] \frac{1}{1-r}\right. \\
& +\frac{\left(\frac{r}{1-r}\right)^{2}}{1-\left(\frac{r}{1-r}\right)^{2}}\left(1+\frac{r}{1-r}+\frac{r}{(1-r)^{2}}\right) \\
& +\frac{2 \frac{r}{1-r}}{\left(1-\left(\frac{r}{1-r}\right)^{2}\right)^{2}}\left[\frac{r}{1-r}+\frac{r^{2}}{(1-r)^{2}}\right] \frac{r}{(1-r)^{2}} \\
& +2 \frac{\left(\frac{r}{1-r}\right)^{2}}{1-\left(\frac{r}{1-r}\right)^{2}}\left(\frac{r}{(1-r)^{2}}+\frac{r^{2}}{(1-r)^{3}}\right) \\
& \left.+\frac{r}{1-r}+\frac{r}{(1-r)^{2}}\right\} .
\end{aligned}
$$

Finally, we can simplify the previous expression and find that

$$
\mathcal{F}(r)=\frac{2 \frac{r}{1-r}}{\left(1-\left(\frac{r}{1-r}\right)^{2}\right)^{2}}\left(\frac{r}{(1-r)^{3}}+\frac{r}{(1-r)^{4}}\right)+\frac{\left(\frac{r}{1-r}\right)^{2}}{1-2 r}
$$




$$
+2 \frac{\left(\frac{r}{1-r}\right)^{2}}{1-\left(\frac{r}{1-r}\right)^{2}} \frac{1}{(1-r)^{3}}+\frac{r}{1-r}+\frac{r}{(1-r)^{2}} .
$$

We observe that $\mathcal{F}$ is a continuous function in a neighborhood of $r=0$ and satisfies $\mathcal{F}(0)=0$. Thus, it exists $0<\mathcal{C}$ such that $\mathcal{F}(\mathcal{C})<1$. We finally observe that if $\left\|v_{0}\right\|_{A^{1}} /\left\langle u_{0}\right\rangle<\mathcal{C}$ this condition propagates in time and ensures the following bound

$$
\|v(t)\|_{A^{1}} \leqslant\left\|v_{0}\right\|_{A^{1}} e^{-\delta t}
$$

for small enough $0<\delta \ll 1$. This last inequality together with a close inspection of the energy estimates in Theorem 1 lead to the following inequality

$$
\frac{d}{d t}\|u\|_{H^{2}}^{2} \leqslant C\left(u_{0}\right)\|u\|_{H^{2}}^{2},
$$

and then we conclude the global bound for the $H^{2}$ norm using Gronwall's inequality.

\section{Proof of Theorem 3}

In this section we prove the existence of global weak solutions for certain initial data satisfying appropriate size restriction in the space $A^{0}$. We emphasize that this space is scale invariant with respect to the scaling of the equation. First we obtain a priori estimates, then we consider a vanishing viscosity approximation and prove the convergence of the approximate solutions.

A priori estimates. Following the previous ideas, the first nonlinear term contributes with

$$
\begin{aligned}
\left\|N L_{1}\right\|_{A^{0}} & =\left\|\sum_{n \in \mathbb{Z}^{+}}(-1)^{n}\left(\mathfrak{S}_{1}\right)^{2 n}\left(\frac{\Lambda v}{\left\langle u_{0}\right\rangle}+\frac{\Lambda v}{\left\langle u_{0}\right\rangle} \sum_{m \in \mathbb{Z}^{+}}(-1)^{m}\left(\frac{v}{\left\langle u_{0}\right\rangle}\right)^{m}\right)\right\|_{A^{0}} \\
& \leqslant \sum_{n \in \mathbb{Z}^{+}}\left(\frac{\|v\|_{A^{0}}}{\left\langle u_{0}\right\rangle-\|v\|_{A^{0}}}\right)^{2 n}\left(\frac{\|v\|_{A^{1}}}{\left\langle u_{0}\right\rangle-\|v\|_{A^{0}}}\right) \\
& \leqslant\left(\frac{\|v\|_{A^{1}}}{\left\langle u_{0}\right\rangle-\|v\|_{A^{0}}}\right)\left(\frac{1}{1-\left(\frac{\|v\|_{A^{0}}}{\left\langle u_{0}\right\rangle-\|v\|_{A^{0}}}\right)^{2}}-1\right) \\
& \leqslant\left(\frac{\|v\|_{A^{1}}}{\left\langle u_{0}\right\rangle-\|v\|_{A^{0}}}\right)\left(\frac{\left(\frac{\|v\|_{A^{0}}}{\left\langle u_{0}\right\rangle-\|v\|_{A^{0}}}\right)^{2}}{1-\left(\frac{\|v\|_{A^{0}}}{\left\langle u_{0}\right\rangle-\|v\|_{A^{0}}}\right)^{2}}\right) .
\end{aligned}
$$

The second nonlinear term can be estimated as

$$
\left\|N L_{2}\right\|_{A^{0}}=\left\|\sum_{n \in \mathbb{Z}^{+}}(-1)^{n}\left(\mathfrak{S}_{1}\right)^{2 n}\left(\frac{H v \partial_{x} v}{\left\langle u_{0}\right\rangle^{2}}+\frac{H v \partial_{x} v}{\left\langle u_{0}\right\rangle^{2}} \sum_{m \in \mathbb{Z}^{+}}(-1)^{m}(1+m)\left(\frac{v}{\left\langle u_{0}\right\rangle}\right)^{m}\right)\right\|_{A^{0}}
$$




$$
\begin{aligned}
& \leqslant \sum_{n \in \mathbb{Z}^{+}}\left(\frac{\|v\|_{A^{0}}}{\left\langle u_{0}\right\rangle-\|v\|_{A^{0}}}\right)^{2 n}\left(\frac{\|v\|_{A^{0}}\|v\|_{A^{1}}}{\left(\left\langle u_{0}\right\rangle-\|v\|_{A^{0}}\right)^{2}}\right) \\
& \leqslant\left(\frac{\|v\|_{A^{0}}\|v\|_{A^{1}}}{\left(\left\langle u_{0}\right\rangle-\|v\|_{A^{0}}\right)^{2}}\right)\left(\frac{\left(\frac{\|v\|_{A^{0}}}{\left\langle u_{0}\right\rangle-\|v\|_{A^{0}}}\right)^{2}}{1-\left(\frac{\|v\|_{A^{0}}}{\left\langle u_{0}\right\rangle-\|v\|_{A^{0}}}\right)^{2}}\right) .
\end{aligned}
$$

Finally, we find that

$$
\begin{gathered}
\left\|N L_{3}\right\|_{A^{0}}=\left\|\Lambda v \frac{1}{\left\langle u_{0}\right\rangle} \sum_{n \in \mathbb{Z}^{+}}(-1)^{n}\left(\frac{v}{\left\langle u_{0}\right\rangle}\right)^{n}\right\|_{A^{0}} \\
\leqslant \frac{\|v\|_{A^{1}}}{\left\langle u_{0}\right\rangle} \sum_{n \in \mathbb{Z}^{+}}\left(\frac{\|v\|_{A^{0}}}{\left\langle u_{0}\right\rangle}\right)^{n} \\
\leqslant\|v\|_{A^{1}}\left(\frac{1}{\left\langle u_{0}\right\rangle-\|v\|_{A^{0}}}-\frac{1}{\left\langle u_{0}\right\rangle}\right) \\
\left\|N L_{4}\right\|_{A^{0}}=\left\|\left(\frac{H v \partial_{x} v}{\left\langle u_{0}\right\rangle^{2}}+\frac{H v \partial_{x} v}{\left\langle u_{0}\right\rangle^{2}} \sum_{n \in \mathbb{Z}^{+}}(-1)^{n}(1+n)\left(\frac{v}{\left\langle u_{0}\right\rangle}\right)^{n}\right)\right\|_{A^{0}} \\
\leqslant \frac{\|v\|_{A^{0}}\|v\|_{A^{1}}}{\left\langle u_{0}\right\rangle^{2}}+\frac{\|v\|_{A^{0}}\|v\|_{A^{1}}}{\left\langle u_{0}\right\rangle^{2}} \sum_{n \in \mathbb{Z}^{+}}(1+n)\left(\frac{\|v\|_{A^{0}}}{\left\langle u_{0}\right\rangle}\right)^{n} \\
\leqslant \frac{\|v\|_{A^{0}}\|v\|_{A^{1}}}{\left(\left\langle u_{0}\right\rangle-\|v\|_{\left.A^{0}\right)^{2}}\right.} .
\end{gathered}
$$

We define

$$
s=\frac{\|v\|_{A^{0}}}{\left\langle u_{0}\right\rangle} .
$$

Collecting the previous estimates, we find that

$$
\begin{aligned}
\frac{d}{d t}\|v\|_{A^{0}}+\frac{\|v\|_{A^{1}}}{\left\langle u_{0}\right\rangle} \leqslant & \frac{\|v\|_{A^{1}}}{\left\langle u_{0}\right\rangle}\left[\frac{s}{(1-s)^{2}}+\frac{s}{1-s}+\left(\frac{s}{(1-s)^{2}}\right)\left(\frac{\left(\frac{s}{1-s}\right)^{2}}{1-\left(\frac{s}{1-s}\right)^{2}}\right)\right. \\
& \left.+\left(\frac{1}{1-s}\right)\left(\frac{\left(\frac{s}{1-s}\right)^{2}}{1-\left(\frac{s}{1-s}\right)^{2}}\right)\right]
\end{aligned}
$$

Using the hypotheses on $\tilde{\mathcal{C}}$, we conclude that

$$
s \leqslant \tilde{\mathcal{C}}
$$

implies

$$
\frac{d}{d t}\|v\|_{A^{0}}+\delta\|v\|_{A^{1}} \leqslant 0
$$

and that, thank to a Poincaré-type inequality, leads to

$$
\|v(t)\|_{L^{\infty}} \leqslant\left\|v_{0}\right\|_{A^{0}} e^{-\delta t}
$$


Furthermore, the solution also enjoys the following parabolic gain of regularity

$$
\int_{0}^{t}\|v(s)\|_{H^{0.5}}^{2} d s \leqslant \int_{0}^{t}\|v(s)\|_{A^{1}} d s \sup _{s}\|v(s)\|_{L^{1}} \leqslant \int_{0}^{t}\|v(s)\|_{A^{1}} d s\left\|v_{0}\right\|_{A^{0}} 2 \pi .
$$

Approximated solutions. To construct the approximate solutions, we consider the following vanishing viscosity approximated problem

$$
\partial_{t} u^{\varepsilon}+\partial_{x} \arctan \left(\frac{H u^{\varepsilon}}{u^{\varepsilon}}\right)=\varepsilon \partial_{x}^{2} u^{\varepsilon} \quad(x, t) \text { on } \mathbb{S}^{1} \times[0, T],
$$

with a mollified initial data

$$
u^{\varepsilon}(x, 0)=\mathcal{M}_{\varepsilon} * u_{0}(x) .
$$

The corresponding approximate solution exists globally and remains smooth.

Compactness. Fix $0<T<\infty$. We have that $u^{\varepsilon}$ is uniformly bounded in

$$
L^{\infty}\left(0, T ; A^{0}\right) \cap L^{2}\left(0, T ; H^{0.5}\right) .
$$

This implies weak-* convergence

$$
u^{\varepsilon} \stackrel{*}{\rightarrow} u,
$$

in

$$
L^{\infty}\left([0, T] \times \mathbb{S}^{1}\right)
$$

and weak convergence

$$
u^{\varepsilon} \rightarrow u,
$$

in

$$
L^{2}\left(0, T ; H^{0.5}\left(\mathbb{S}^{1}\right)\right) .
$$

Furthermore, $\partial_{t} u^{\epsilon}$ is uniformly bounded in

$$
L^{2}\left(0, T ; H^{-1.5}\right) .
$$

A standard application of Aubin-Lions Theorem [32] ensures the strong convergence (after maybe taking a subsequence)

$$
u^{\varepsilon} \rightarrow u, H u^{\varepsilon} \rightarrow H u
$$

in

$$
L^{2}\left(0, T ; L^{2}\right) .
$$

Taking another subsequence if necessary, we obtain that

$$
u^{\varepsilon}(x, t) \rightarrow u(x, t) \text { a.e in } \mathbb{S}^{1} \times[0, T]
$$

In particular, we conclude the lower bound

$$
\min _{x} u_{0}(x) \leqslant u(x, t) \text { a.e in } \mathbb{S}^{1} \times[0, T] .
$$


Passing to the limit. Being the other terms linear, we only have to take into consideration the convergence of

$$
J=\int_{0}^{T} \int_{\mathbb{S}^{1}}\left(\arctan \left(\frac{H u^{\varepsilon}}{u^{\varepsilon}}\right)-\arctan \left(\frac{H u}{u}\right)\right) \partial_{x} \phi d x d s .
$$

We have that

$$
J \leqslant \int_{0}^{T} \int_{\mathbb{S}^{1}}\left|\frac{H u^{\varepsilon}}{u^{\varepsilon}}-\frac{H u}{u}\right|\left|\partial_{x} \phi\right| d x d s .
$$

Using the lower bounds for $u$ and $u^{\epsilon}$ together with Hölder inequality, we conclude that

$$
J \rightarrow 0 \text {. }
$$

This concludes the proof of the existence of a global weak solution $u$.

\section{LinK BeTWEen (1) AND (4)}

We now look for a solution of (1) having the following form

$$
u(x, t)=\left\langle u_{0}\right\rangle+\varepsilon \sum_{j=0}^{\infty} \varepsilon^{j} f^{(j)}(x, t),
$$

(here $\varepsilon$ can be though as the displacement from the homogeneous state $\left\langle u_{0}\right\rangle$ ). The idea is to truncate the series up to certain order, two say,

$$
f(x, t)=\varepsilon f^{(0)}(x, t)+\varepsilon^{2} f^{(1)}(x, t),
$$

and see what $f$ solves. In this way we will obtain that (up to $O\left(\varepsilon^{3}\right)$ ), $f$ solves (4). A similar approach has been used in the study of free boundary problems for incompressible fluids (see [7, 20, 21] and the references therein). First, we observe that (1) can be equivalently written as

$$
\partial_{t} u+\frac{u \Lambda u-H u \partial_{x} u}{u^{2}+(H u)^{2}}=0 \quad(x, t) \text { on } \mathbb{S}^{1} \times[0, T] .
$$

Thus,

$$
\partial_{t} u\left(\left\langle u_{0}\right\rangle^{2}+2\left(u-\left\langle u_{0}\right\rangle\right)\left\langle u_{0}\right\rangle+\left(u-\left\langle u_{0}\right\rangle\right)^{2}+(H u)^{2}\right)+u \Lambda u-H u \partial_{x} u=0 .
$$

Forcing the previous ansatz and matching the powers of $\varepsilon$, we find that $f^{(0)}$ solves

$$
\partial_{t} f^{(0)}+\frac{\Lambda f^{(0)}}{\left\langle u_{0}\right\rangle}=0
$$

Similarly, $f^{(1)}$ solves

$$
\partial_{t} f^{(1)}\left\langle u_{0}\right\rangle^{2}+2 \partial_{t} f^{(0)} f^{(0)}\left\langle u_{0}\right\rangle+\left\langle u_{0}\right\rangle \Lambda f^{(1)}+f^{(0)} \Lambda f^{(0)}-H f^{(0)} \partial_{x} f^{(0)}=0 .
$$

Thus, substituting $\left\langle u_{0}\right\rangle \partial_{t} f^{(0)}$ by $-\Lambda f^{(0)}$, we find that $f$ solves

$$
\partial_{t} f+\frac{1}{\left\langle u_{0}\right\rangle} \Lambda f-\frac{1}{\left\langle u_{0}\right\rangle^{2}} \partial_{x}(H f f)=O\left(\varepsilon^{3}\right)
$$


Thus, neglecting the $O\left(\varepsilon^{3}\right)$ terms we find that

$$
g(x, t)=\frac{f\left(x, t\left\langle u_{0}\right\rangle\right)}{\left\langle u_{0}\right\rangle}
$$

solves (4).

Acknowledgements. R. G-B has been funded by the grant MTM201789976-P from the Spanish government.

\section{REFERENCES}

[1] Hantaek Bae and Rafael Granero-Belinchón. Global existence for some transport equations with nonlocal velocity. Adv. Math., 269:197-219, 2015.

[2] Gregory R Baker, Xiao Li, and Anne C Morlet. Analytic structure of two 1d-transport equations with nonlocal fluxes. Physica D: Nonlinear Phenomena, 91(4):349-375, 1996.

[3] Jan Burczak and Rafael Granero-Belinchón. Boundedness of large-time solutions to a chemotaxis model with nonlocal and semilinear flux. Topol. Methods Nonlinear Anal., 47(1):369-387, 2016.

[4] A. Castro and D. Córdoba. Global existence, singularities and ill-posedness for a nonlocal flux. Adv. Math., 219(6):1916-1936, 2008.

[5] A. Castro and D. Córdoba. Infinite energy solutions of the surface quasi-geostrophic equation. Adv. Math., 225(4):1820-1829, 2010.

[6] Dongho Chae, Antonio Córdoba, Diego Córdoba, and Marco A. Fontelos. Finite time singularities in a 1D model of the quasi-geostrophic equation. Adv. Math., 194(1):203223, 2005.

[7] CH Cheng, Rafael Granero-Belinchón, Steve Shkoller, and Jon Wilkening. Rigorous asymptotic models of water waves. arXiv preprint arXiv:180\%.001\%6, 2018.

[8] P. Constantin, P. D. Lax, and A. Majda. A simple one-dimensional model for the three-dimensional vorticity equation. Comm. Pure Appl. Math., 38(6):715-724, 1985.

[9] Peter Constantin and Vlad Vicol. Nonlinear maximum principles for dissipative linear nonlocal operators and applications. Geom. Funct. Anal., 22(5):1289-1321, 2012.

[10] Antonio Córdoba and Diego Córdoba. A pointwise estimate for fractionary derivatives with applications to partial differential equations. Proc. Natl. Acad. Sci. USA, 100(26):15316-15317, 2003.

[11] Antonio Córdoba and Diego Córdoba. A maximum principle applied to quasigeostrophic equations. Comm. Math. Phys., 249(3):511-528, 2004.

[12] Antonio Córdoba, Diego Córdoba, and Marco A. Fontelos. Formation of singularities for a transport equation with nonlocal velocity. Ann. of Math. (2), 162(3):1377-1389, 2005.

[13] Tam Do. On a 1D transport equation with nonlocal velocity and supercritical dissipation. J. Differential Equations, 256(9):3166-3178, 2014.

[14] Tam Do, Vu Hoang, Maria Radosz, and Xiaoqian Xu. One-dimensional model equations for hyperbolic fluid flow. Nonlinear Anal., 140:1-11, 2016.

[15] Hongjie Dong. Well-posedness for a transport equation with nonlocal velocity. $J$. Funct. Anal., 255(11):3070-3097, 2008.

[16] Rafael Granero-Belinchón. Global existence for the confined Muskat problem. SIAM Journal on Mathematical Analysis, 46(2):1651-1680, 2014.

[17] Rafael Granero-Belinchón. On the fractional fisher information with applications to a hyperbolic-parabolic system of chemotaxis. Journal of Differential Equations, 262(4):3250-3283, 2017. 
[18] Rafael Granero-Belinchón and Martina Magliocca. Global existence and decay to equilibrium for some crystal surface models. To appear in Discrete and Continuous Dynamical Systems-A, arXiv preprint arXiv:1804.09645, 2018.

[19] Rafael Granero-Belinchón and Rafael Orive-Illera. An aggregation equation with a nonlocal flux. Nonlinear Anal., 108:260-274, 2014.

[20] Rafael Granero-Belinchón and Stefano Scrobogna. Asymptotic models for free boundary flow in porous media. arXiv preprint arXiv:1810.10756, 2018.

[21] Rafael Granero-Belinchón and Stefano Scrobogna. On an asymptotic model for free boundary Darcy flow in porous media. arXiv preprint arXiv:1810.11798, 2018.

[22] $\mathrm{Vu}$ Hoang and Maria Radosz. Cusp formation for a nonlocal evolution equation. Arch. Ration. Mech. Anal., 224(3):1021-1036, 2017.

[23] Omar Lazar. On a 1D nonlocal transport equation with nonlocal velocity and subcritical or supercritical diffusion. J. Differential Equations, 261(9):4974-4996, 2016.

[24] Omar Lazar and Pierre-Gilles Lemarié-Rieusset. Infinite energy solutions for a 1D transport equation with nonlocal velocity. Dyn. Partial Differ. Equ., 13(2):107-131, 2016.

[25] Dong Li and Jose Rodrigo. Blow-up of solutions for a 1D transport equation with nonlocal velocity and supercritical dissipation. Adv. Math., 217(6):2563-2568, 2008.

[26] Andrew J Majda and Andrea L Bertozzi. Vorticity and incompressible flow, volume 27. Cambridge University Press, 2002.

[27] Yoshimasa Matsuno. Linearization of novel nonlinear diffusion equations with the hilbert kernel and their exact solutions. Journal of mathematical physics, 32(1):120$126,1991$.

[28] Hisashi Okamoto, Takashi Sakajo, and Marcus Wunsch. On a generalization of the Constantin-Lax-Majda equation. Nonlinearity, 21(10):2447-2461, 2008.

[29] Diana Stan, Félix del Teso, and Juan Luis Vázquez. Finite and infinite speed of propagation for porous medium equations with fractional pressure. Comptes Rendus Mathematique, 352(2):123-128, 2014.

[30] Diana Stan, Félix del Teso, and Juan Luis Vázquez. Finite and infinite speed of propagation for porous medium equations with nonlocal pressure. Journal of Differential Equations, 260(2):1154-1199, 2016.

[31] Stefan Steinerberger. A nonlocal transport equation describing roots of polynomials under differentiation. arXiv preprint arXiv:1811.04844, 2018.

[32] Roger Temam. Navier-Stokes equations: theory and numerical analysis, volume 343. American Mathematical Soc., 2001.

E-mail address: rafael.granero@unican.es

Departamento de Matemáticas, Estadística y Computación, Universidad de Cantabria. Avda. Los Castros s/n, Santander, Spain. 